Annals of Pure and Applied Mathematics

Vol. 13, No. 1, 2017, 119-124

ISSN: 2279-087X (P), 2279-0888(online)

Published on 3 March 2017

Annals of

www.researchmathsci.org

DOI: http://dx.doi.org/10.22457/apam.v13n1a11

Pure and Applied Mathematics

\title{
Some Relations Related to Centralizers on Semiprime Semiring
}

\author{
D.Mary Florence $^{1}$, R.Murugesan ${ }^{2}$ and P.Namasivayam ${ }^{3}$
}

\author{
${ }^{1}$ Department of Mathematics, Kanyakumari Community College \\ Mariagiri - 629153, Tamil Nadu, India. E-mail: dmaryflorence@gmail.com \\ ${ }^{2}$ Department of Mathematics, Thiruvalluvar College \\ Papanasam - 627425, Tamil Nadu, India.E-mail: rmurugesa2020@yahoo.com \\ ${ }^{3}$ Department of Mathematics, The M.D.T Hindu College \\ Tirunelveli - 627010, Tamil Nadu, India.E-mail: vasuhe2010@ gmail.com
}

Received 8 February 2017; accepted 28 February 2017

Abstract. In this paper, we generalize the following result. If $S$ is a 2-torsion free semiprime semiring and $T: S \rightarrow S$ be an additive mapping such that $2 T(x y x)=$ $T(x) y x+x y T(x)$ holds for all $x, y \in S$, then $T$ is a centralizer.

Keywords: Semiring, semiprime semiring, centralizer, jordan centralizer, left (right) centralizer.

AMS Mathematics Subject Classification (2010): 16Y60, 16N60

\section{Introduction}

Semirings has been formally introduced by Vandiver in 1934. Golan [9] discussed the notion of semirings and their applications. In [4], Chandramouleeswaran and Tiruveni worked on the derivations on semirings. Zalar [1] studied centralizers on semiprime rings and proved that Jordan centralizer and centralizers of this rings coincide. In [15], Vukman and Irena proved that if $R$ is a 2-torsion free semiprime ring and $T: R \rightarrow R$ is an additive mapping such that $2 T(x y x)=T(x) y x+x y T(x)$ holds for all $x, y \in R$, then $T$ is a centralizer. In papers $[6,7,8]$ the authors Hoque and Paul worked on centralizers on semiprime Gamma rings and developed the results of [15] in Gamma rings. Motivated by this Florence and Murugesan [10] studied the notion of semirings and proved that Jordan centralizer of a 2-torsion free semiprime semiring is a centralizer. Here we develop the results of [7,15] in semirings by assuming that $S$ be a 2-torsion free semiprime semiring and $T: S \rightarrow S$ be an additive mappingsuch that $2 T(x y x)=$ $T(x) y x+x y T(x)$ holds for all $x, y \in S$. Then $T$ is a centralizer.

Now we recall the following definitions and results:

Let $S$ be a non empty set followed with two binary operation ' + ' and '.' such that

i) $\quad(S,+)$ is a commutative monoid with identity element 0 .

ii) $\quad(S,$.$) is a monoid with identity element 1$.

iii) Multiplication distributes over addition from either side.

That is, $a \cdot(b+c)=a \cdot b+a \cdot c$,

$(b+c) \cdot a=b \cdot a+c \cdot a$. Then $S$ is called a semiring. 
D.Mary Florence, R.Murugesan and P.Namasivayam

A Semiring $S$ is prime if $x S y=0$ implies $x=0$ or $y=0 \forall x, y \in S$, and semiprime if $x S x=0$ implies $x=0 \forall x \in S$. A semiring $S$ is 2-torsion free if $2 x=0$, $x \in S \Rightarrow x=0$. As usual the commutator $x y-y x$ will be denoted by $[x, y]$.More over the $\operatorname{set} Z(S)=\{x \in S: x y=y x \forall y \in S\}$. we shall use basic commutator identities $[x, y z]=$ $[x, y] z+y[x, z]$ and $[x z, y]=[x, y] z+x[z, y]$.An additive mapping $T: S \rightarrow S$ is called a Left (Right) Centralizer if $T(x y)=T(x) y \quad((T(x y)=x T(y))$ holds for all $x, y \in S$. We call $T$ is a centralizer whichis both left and right centralizer. For a fixed $a \in S$ then $T(x)=a x$ is a left centralizer and $T(x)=x a$ is a right centralizer. An additive mapping $T: S \rightarrow S$ is called a left (right) Jordan centralizer if $T(x x)=T(x) x(T(x x)=x T(x))$ holds for all $x \in S$. Every left centralizer is a Jordan left centralizer but the converse is not in general true. An additive mapping $T: S \rightarrow S$ is a Jordan centralizer if $T(x y+y x)=$ $T(x) y+y T(x)$ for all,$y \in S$. Every centralizer is a Jordan centralizer but Jordan centralizer is not in general a centralizer.

Let $(S,+,$.$) be a semiring. An element a of S$ is called additively left cacellative if for all $a, b, c \in S, a+b=a+c \Rightarrow b=c$. If every element of a semiring $S$ is additively left cancellative, it is called an additively left cancellative semiring. If every element of a semiring $S$ is additively right cancellative, it is called additively right cancellative semiring. Similarly we can define multiplicatively left and right cancellative semiring.

\section{The centralizers of semiprime semiring}

Lemma 2.1. Let $S$ be a semiprime semiring. Suppose that the relation

$a x b+b x c=0 \forall x \in S$ and some $a, b \in S$. In this case $(a+c) x b=0, \forall x \in S$

Proof: By hypothesis we have $a x b+b x c=0$

Putting $x=x b y$ yields $a x b y b+b x b y c=0$

On the other hand right multiplying (1) by $y b$ we get

$$
a x b y b+b x c y b=0 \quad \forall x, y \in S \text {. }
$$

Subtracting (3) from (2) we obtainaxbyb + bxbyc $-a x b y b-b x c y b=0$

$$
\begin{aligned}
& b x b y c-b x c y b=0 \\
& b x(b y c-c y b)=0
\end{aligned}
$$

Putting $x=y c x$ in (4) we get

$$
\text { bycx }(b y c-c y b)=0
$$

Left multiplying (4) by $c y$ we obtain

$$
c y b x(b y c-c y b)=0
$$

Subtracting (6) from (5) yields

$$
\begin{aligned}
& \text { bycx }(b y c-c y b)-c y b x(b y c-c y b)=0 \\
& (b y c-c y b) x(b y c-c y b)=0
\end{aligned}
$$

Since $S$ is semiprime, $b y c-c y b=0$

$$
\Rightarrow b y c=c y b, y \in S
$$

Replace $y=x$, in the above relation we get, $b x c=c x b$

So (1) becomes $a x b+c x b=0$

$$
(a+c) x b=0, \forall x \in S
$$

Hence the proof is complete.

Lemma 2.2. Let $S$ be a 2-torsion free semiprime semiring and let $T: S \rightarrow S$ be an additive mapping such that $2 T(x y x)=T(x) y x+x y T(x)$ holds for all $x, y \in S$. Then $2 T(x x)=$ $T(x) x+x T(x)$.

Proof:

By the assumption we have $\quad 2 T(x y x)=T(x) y x+x y T(x)$ 
Some Relations related to Centralizers on Semiprime Semiring

Linearizing the above by putting $x+z$ for $x$ we obtain

$2 T((x+z) y(x+z))=T(x+z) y(x+z)+(x+z) y T(x+z)$

$$
2 T(x y z+z y x)=T(x) y z+T(z) y x+x y T(z)+z y T(x)
$$

Substituting $z=x^{2}$ the relation (8) yields

$$
2 T\left(x y x^{2}+x^{2} y x\right)=T(x) y x^{2}+T\left(x^{2}\right) y x+x y T\left(x^{2}\right)+x^{2} y T(x)
$$

Substitution for $y=x y+y x$ in (7) we arrive at

$$
\begin{aligned}
& 2 T(x(x y+y x) x)=T(x)(x y+y x) x+x(x y+y x) T(x) \\
& 2 T\left(x^{2} y x+x y x^{2}\right)=T(x) x y x+T(x) y x^{2}+x^{2} y T(x)+x y x T(x)
\end{aligned}
$$

Subtracting (10) from (9) we get

$$
\begin{aligned}
& T\left(x^{2}\right) y x+x y T\left(x^{2}\right)-T(x) x y x-x y x T(x)=0 \\
& \left(T\left(x^{2}\right)-T(x) x\right) y x+x y\left(T\left(x^{2}\right)-x T(x)\right)=0
\end{aligned}
$$

From the above relation taking

$$
a=T\left(x^{2}\right)-T(x) x, x=y, b=x, c=T\left(x^{2}\right)-x T(x)
$$

Now applying lemma 2.1 follows that

$$
\begin{aligned}
& \left(T\left(x^{2}\right)-T(x) x+T\left(x^{2}\right)-x T(x)\right) y x=0 \\
& \left(2 T\left(x^{2}\right)-T(x) x-x T(x)\right) y x=0
\end{aligned}
$$

Taking $A(x)=2 T\left(x^{2}\right)-T(x) x-x T(x)$, then the above relation becomes,

$$
A(x) y x=0
$$

Applying $y=x y A(x)$ in (11) gives $A(x) x y A(x) x=0$

By the semiprimeness of $S, \quad A(x) x=0$

On the other hand left multiplying (11) by $x$ and right multiplying by $A(x)$ we obtain

$$
x A(x) y x A(x)=0
$$

Since $S$ is semiprime, $\quad x A(x)=0$

Putting $x+y$ for $x$ in (12) we get

$A(x+y)(x+y)=0$

$A(x) y+A(y) x+B(x, y) x+B(x, y) y+A(x) x+A(y) y=0$ where

$B(x, y)=2 T(x y+y x)-T(x) y-T(y) x-x T(y)-y(T(x)$

Using (12) the above relation reduces to

$$
\begin{aligned}
& A(x) y+A(y) x+B(x, y) x+B(x, y) y \\
& A(x) y+A(y) x+B(x, y)(x+y)
\end{aligned}
$$

Replacing $x+y=x$

$$
A(x) y+A(y) x+B(x, y) x \text {. }
$$

Right multiplication of the above relation by $A(x)$ gives because of (13)

$$
\begin{aligned}
& A(x) y A(x)+A(y) x A(x)+B(x, y) x A(x) \\
& A(x) y A(x)=0 \forall x, y \in S
\end{aligned}
$$

By the semiprimeness of $S$, we get $A(x)=0$.

Thus $2 T\left(x^{2}\right)-T(x) x-x T(x)=0$

$$
2 T\left(x^{2}\right)=T(x) x+x T(x)
$$

This completes the proof.

Lemma 2.3. Let $S$ be a 2-torsion free semiprime semiring and let $T: S \rightarrow S$ be an additive mapping, suppose that $2 T(x y x)=T(x) y x+x y T(x)$ holds for all pairs $x, y \in S$. Then $[T(x), x]=0$

Proof: We have $2 T(x x)=T(x) x+x T(x)$

Linearizing the above by replacing $x=x+y$ we obtain

$2 T(x y+y x)=T(x) y+T(y) x+x T(y)+y T(x)$

Replacing $y=2 x y x$ in (15) and using the assumption of the theorem yields

$4 T\left(x^{2} y x+x y x^{2}\right)=2 T(x) x y x+2 T(x y x) x+x 2 T(x y x)+2 x y x T(x)$ 
D.Mary Florence, R.Murugesan and P.Namasivayam

$$
\begin{aligned}
= & 2 T(x) x y x+(T(x) y x+x y T(x)) x+x(T(x) y x \\
& +x y T(x))+2 x y x T(x) \\
2\left(2 T\left(x^{2} y x+x y x^{2}\right)\right) & =2 T(x) x y x+T(x) y x^{2}+x y T(x) x+x T(x) y x \\
& +x^{2} y T(x)+2 x y x T(x)
\end{aligned}
$$

Applying (10) in (16) gives

$$
\begin{gathered}
2\left(T(x) x y x+T(x) y x^{2}+x^{2} y T(x)+x y x T(x)\right)=2 T(x) x y x+T(x) y x^{2} \\
+x y T(x) x+x T(x) y x+x^{2} y T(x)+2 x y x T(x) \\
T(x) y x^{2}+x^{2} y T(x)-x y T(x) x-x T(x) y x=0
\end{gathered}
$$

Replacing $y=y x$ in (17) we arrive at

$$
T(x) y x^{3}+x^{2} y x T(x)-x y x T(x) x-x T(x) y x^{2}=0 \forall x, y \in S
$$

Right multiplication of (17) by $x$ yields,

$$
T(x) y x^{3}+x^{2} y T(x) x-x y T(x) x^{2}-x T(x) y x^{2}=0
$$

Subtracting (18) from (19) gives

$$
\begin{aligned}
& x^{2} y T(x) x-x y T(x) x^{2}-x^{2} y x T(x)+x y x T(x) x=0 \\
& x^{2} y[T(x) x-x T(x)]-x y[T(x) x-x T(x)] x=0 \\
& x^{2} y[T(x), x]-x y[T(x), x] x=0
\end{aligned}
$$

Applying $y=T(x) y$ in (20) leads to

$$
x^{2} T(x) y[T(x), x]-x T(x) y[T(x), x] x=0
$$

Left multiplication of (20) by $T(x)$ gives

$$
T(x) x^{2} y[T(x), x]-T(x) x y[T(x), x] x=0
$$

Subtracting (21) from (22) we obtain

$T(x) x^{2} y[T(x), x]-T(x) x y[T(x), x] x-x^{2} T(x) y[T(x), x]+x T(x) y[T(x), x] x=0$

$\left[T(x) x^{2}-x^{2} T(x)\right] y[T(x), x]+[x T(x)-T(x) x] y[T(x), x] x=0$

$[T(x), x] x y[T(x), x]+x[T(x), x] y[T(x), x]+[x, T(x)] y[T(x), x] x=0$

From (20) we can write $x y[T(x), x]=y[T(x), x] x$ and using $[x, T(x)]=-[T(x), x]$ the above relation becomes

$[T(x), x] y[T(x), x] x+x[T(x), x] y[T(x), x]-[T(x), x] y[T(x), x] x=0$

$x[T(x), x] y[T(x), x]=0 \forall x, y \in S$

Substituting $y=y x$ in the above relation

$x[T(x), x] y x[T(x), x]=0 \forall x, y \in S$

By the semiprimeness of $S, \quad x[T(x), x]=0$

Replacing $y=x y$ in (17) gives

$$
\begin{aligned}
& T(x) x y x^{2}+x^{2} x y T(x)-x x y T(x) x-x T(x) x y x=0 \\
& T(x) x y x^{2}+x^{3} y T(x)-x^{2} y T(x) x-x T(x) x y x=0
\end{aligned}
$$

Left multiplication of (17) by $x$ we get

$$
x T(x) y x^{2}+x^{3} y T(x)-x^{2} y T(x) x-x^{2} T(x) y x=0
$$

Subtracting (25) from (24) we obtain

$$
\begin{aligned}
& {[T(x) x-x T(x)] y x^{2}-x[T(x) x-x T(x)] y x=0} \\
& {[T(x), x] y x^{2}-x[T(x), x] y x=0}
\end{aligned}
$$

Using (23) in the above relation yields $[T(x), x] y x^{2}=0$

Applying $y T(x)$ for $y$ in (26) we obtain $[T(x), x] y T(x) x^{2}=0$

Right multiplication of (26) by $T(x)$ gives $[T(x), x] y x^{2} T(x)=0$

Subtracting (28) from (27) we get

$$
\begin{aligned}
& {[T(x), x] y\left(T(x) x^{2}-x^{2} T(x)\right)=0} \\
& {[T(x), x] y\left[T(x), x^{2}\right]=0} \\
& {[T(x), x] y([T(x), x] x+x[T(x), x])=0}
\end{aligned}
$$

Using (23) in the above relation reduces to

$$
[T(x), x] y[T(x), x] x=0
$$


Some Relations related to Centralizers on Semiprime Semiring

Putting $y=x y$ in the above implies $[T(x), x] x y[T(x), x] x=0$

Since $S$ is semiprime $\quad[T(x), x] x=0$

Putting $x=x+y$ in (23) yields

$(x+y)[T(x+y), x+y]=0$

$x[T(x), x]+x[T(x), y]+x[T(y), x]+x[T(y), y]+y[T(x), x]+y[T(x), y]$

$$
+y[T(y), x]+y[T(y), y]=0
$$

Using (23) the aboverelation reduces to

$x[T(x), y]+x[T(y), x]+x[T(y), y]+y[T(x), x]+y[T(x), y]+y[T(y), x]=0$

$(x+y)[T(x), y]+(x+y)[T(y), x]+x[T(y), y]+y[T(x), x]=0$

Now replacing $x+y=x$ gives

$x[T(x), y]+x[T(y), x]+x[T(y), y]+y[T(x), x]=0$

Left multiplication of (30) by $[T(x), x]$ we obtain

$[T(x), x] x[T(x), y]+[T(x), x] x[T(y), x]+[T(x), x] x[T(y), y]$

$+[T(x), x] y[T(x), x]=0$

Using (29) the above relation reduces to $[T(x), x] y[T(x), x]=0$

Since $S$ is semiprime $[T(x), x]=0$.

Theorem 2.1. Let $S$ be a 2-torsion free semiprime semiring. Let $T: S \rightarrow S$ be an additive mapping, suppose that $2 T(x y x)=T(x) y x+x y T(x)$ holds for all $x, y \in S$. Then $T$ is a centralizer.

Proof: We have $[T(x), x]=0$

$$
\begin{aligned}
& \Rightarrow T(x) x-x T(x)=0 \\
& \Rightarrow T(x) x=x T(x)
\end{aligned}
$$

Applying the above results in (14) we obtain $2 T\left(x^{2}\right)=2 T(x) x$

Since $S$ is 2-torsion free semiprime semiring $T\left(x^{2}\right)-T(x) x=0$

$$
\Rightarrow T\left(x^{2}\right)=T(x) x
$$

Similarly $T\left(x^{2}\right)=x T(x)$. This means that $T$ is a left and right Jordan Centralizer. By theorem (3.1) in [10] yields that $T$ is a left and right centralizer. Thus the proof is completed.

\section{REFERENCES}

1. B.Zalar, On centralizers of Semiprime rings,Commentationes Mathematicae Universitatis Carolinae, 32(4) (1991) 609-614.

2. M.Bresar, Jordan derivations on semiprime rings, Proc. Amer. Math. Soc., 104 (1988) 1003-1006.

3. M.Bresar and J.Vukman, On some additive mapping in rings with involution, Aequationes Math., 38 (1989) 178-185.

4. T.Chandramouleeswaran, On derivations of semirings, Advances in Algebra, 3(1) (2010) 123-131.

5. I.N.Herstein, Topics in ring theory, University of Chicago Press (1969).

6. M.F.Hoque and A.C.Paul, Centralizers on semiprime gamma rings, Italian Journal of Pure and Applied Mathematics, 30 (2013) 289-302.

7. M.F. Hoque and A.C.Paul, An equation related to centralizers in semiprime gamma rings, Annals of Pure and Applied Mathematics, 1 (1) (2012) 84-90.

8. M.F.Hoque and A.C.Paul, On centralizers of semiprime gamma rings, International Mathematical Forum, 6(13) (2011) 627-638.

9. J.S.Golan, Semirings and their Applications, Kluwer Academic Press(1969).

10. D.M.Florence, R.Murugesan and P.Namasivayam, Centralizers on semiprime semiring, IOSR Journal of Mathematics, 12 (2016) 86-93. 
D.Mary Florence, R.Murugesan and P.Namasivayam

11. K.Ray Chowdhury, A.Sultana, N.K.Mitra and A.F.M.K.Khan, Some structural properties of semirings, Annals of Pure and Applied Mathematics, 5(2) (2014) 158167.

12. T.Vasanthi and N.Sulochana, On the additive and multiplicative structure of semirings, Annals of Pure and Applied Mathematics, 3(1) (2013) 78-84.

13. J.Vukman, Centralizers on semiprime rings, Comment Math. Univ. Carolinae, 42(2) (2001) 237-245.

14. J.Vukman, An identity related to centralizers in semiprime rings, Comment. Math. Univ. Carolinae, 40 (3) (1999) 447-456.

15. J.Vukman and I.Kosi-ulbl, On centralizers of semiprime rings, Aequationes Math. 66 (3) (2003) 277-283. 